\title{
离子交换和热处理对贵金属掺杂硅酸盐玻璃光致发光的影响
}

\author{
杨修春 \\ (同济大学, 材料科学与工程学院, 先进土木工程材料教育部重点实验室, 上海 201804)
}

摘 要: 贵金属纳米颗粒-玻璃复合材料是一种重要的非线性材料。利用光致发光谱及配位场理论, 深入研究了离子 交换及氢气热处理对硅酸盐玻璃中贵金属离子的引入、还原、成核及生长过程的影响, 发现延长离子交换时间有利 于提高玻璃中金属离子的浓度。对于掺 $\mathrm{Ag}^{+}$硅酸盐玻璃，不仅存在孤立 $\mathrm{Ag}^{+}$离子，同时也存在 $\mathrm{Ag}_{2}{ }^{+}$团簇。 $\mathrm{H}_{2}$ 中热处 理后, 样品中孤立 $\mathrm{Ag}^{+}$离子浓度迅速降低, 同时出现 $\mathrm{Ag}_{3}^{2+}$ 团簇。对于掺 $\mathrm{Cu}^{+}$硅酸盐玻璃, 仅存在孤立 $\mathrm{Cu}^{+}$和 $\mathrm{Cu}^{2+}$, 没 有发现 $\mathrm{Cu}^{+}$团簇的发光峰, $\mathrm{Cu}^{2+}$ 的存在造成 $\mathrm{Cu}^{+}$的发光强度显著降低。掺 $\mathrm{Ag}^{+}$硅酸盐玻璃经 $\mathrm{H}_{2}$ 热处理后，再经过第 二次离子交换往玻璃中掺 $\mathrm{Cu}^{+}$是十分困难的。

关 键 词: 离子交换; 热处理; 贵金属离子及团簇; 光致发光; 配位场理论

中图分类号: TQ174 文献标识码: A

\section{Influences of Ion Exchange and Thermal Treatment on Photoluminescence of Noble Metal Doped Silicate Glasses}

\section{YANG Xiu-Chun}

(Key Laboratory of Advanced Civil Engineering Materials, Ministry of Education, School of Materials Science and Engineering, Tongji University, Shanghai 201804, China)

\begin{abstract}
Noble metallic nanoparticles-glass composites have attracted much interest because of their technical potential as nonlinear media. In present work, the influences of ion-exchange and thermal treatment on the photoluminescence of noble metal doped silicate glasses were discussed based on photoluminescence (PL) spectroscopy and ligand field theory. The results indicate that metallic ion concentration in silicate glass increases with extending ion-exchanged period. There exist isolated $\mathrm{Ag}^{+}$ions and $\mathrm{Ag}_{2}{ }^{+}$clusters in Ag-doped silicate glass. Concentration of the isolated $\mathrm{Ag}^{+}$ions decreases quickly and $\mathrm{Ag}_{3}{ }^{2+}$ clusters appear after annealing the Ag-doped silicate glass in $\mathrm{H}_{2}$. While there exist only isolated $\mathrm{Cu}^{+}$and $\mathrm{Cu}^{2+}$ ions in the $\mathrm{Cu}$-doped silicate glass, no photoluminescence of $\mathrm{Cu}^{+}$clusters are found in the glass. The existence of $\mathrm{Cu}^{2+}$ ions makes the luminescence of $\mathrm{Cu}^{+}$quenching. It is very difficult to introduce $\mathrm{Cu}^{+}$ions into the annealed Ag-doped glass in $\mathrm{H}_{2}$ via $\mathrm{Cu}^{+}$for $\mathrm{Na}^{+}$ion-exchange.
\end{abstract}

Key words: ion-exchange; thermal treatment; nobel metallic ions and clusters; photoluminescence; ligand field theory

早在罗马时代，罗马人就将金属纳米颗粒作 为颜料掺杂于玻璃中, 制备出莱克格斯(Lycurgus) 杯。该杯至今仍保存在大英博物馆中, 在反射光下,
该玻璃奖杯为绿色，在透视光下，该玻璃奖杯为红 色 $^{[1]}$ 。1 857 年, Michal Faraday ${ }^{[2]}$ 系统地研究了金 胶体的制备及其与颜色之间的关系。贵金属纳米颗 
粒具有不同于体材料的表面等离子体共振(SPR)特 性, 可应用于表面增强谱学、化学和生物敏感器、 光能传送器和近场扫描光学显微学等领域 ${ }^{[3-7]}$ 。近 年来, 随着非线性光学的发展, 人们期望制备出非 线性光学性能优良的材料, 用于光存储、传输和全 光开关等器件 ${ }^{[8-15]}$ 。选用玻璃作为基质有很多优点, 如玻璃在大部分波段透明, 具有较好的化学稳定 性和热稳定性, 易于成纤成膜和光学加工等。制备 金属纳米颗粒复合玻璃的方法主要有熔融法、离子 交换法、离子注人法、溶胶一凝胶法、还原气氛处 理法离子或激光辐射法和共溅射法, 其中离子交 换结合热处理法是一种简单而有效的制备方法。通 过离子交换 将金属离子引入钠钙硅酸盐玻璃, 并 通过控制后处理工艺条件, 将金属离子还原成金 属原子，金属原子在合适的条件下成核和生长，可 以原位制备出金属纳米颗粒一玻璃复合材料。研究 表明, 金属纳米颗粒一玻璃复合材料的光学性能随 颗粒的尺寸、浓度、微结构、形状以及界面结构而 变化 ${ }^{[16-25]}$ 。本工作利用光致发光谱和配位场理论研 究了制备工艺对金属离子的引入、还原、成核及生 长过程的影响, 目前这方面的研究还相当缺乏, 对 此深入研究十分必要。

\section{1 实验方法}

采用商用钠钙玻璃 $(25.4 \mathrm{~mm} \times 76.2 \mathrm{~mm} \times 1 \mathrm{~mm})$ 作为玻璃基体, 转变点温度为 $580^{\circ} \mathrm{C}$, 其化学组成为 (以质量计) $70.5 \mathrm{SiO}_{2}-15.5 \mathrm{Na}_{2} \mathrm{O}-7.0 \mathrm{CaO}-4.2 \mathrm{MgO}-1.7 \mathrm{Al}_{2} \mathrm{O}_{3}$ $0.3 \mathrm{~K}_{2} \mathrm{O}-0.3 \mathrm{SO}_{3}-0.06 \mathrm{Fe}_{2} \mathrm{O}_{3}$ 。

钠钙硅酸盐玻璃在 $4500^{\circ} \mathrm{C} \mathrm{CuCl}$ 熔盐中经过离 子交换 $1 \mathrm{~h}$ 和 $3 \mathrm{~h}$ 得到样品 $\mathrm{Cu}-1$ 和 $\mathrm{Cu}-2$, 钠钙硅酸 盐玻璃在 $500^{\circ} \mathrm{C} \mathrm{CuCl}$ 熔盐中离子交换 $3 \mathrm{~h}$ 得到样品

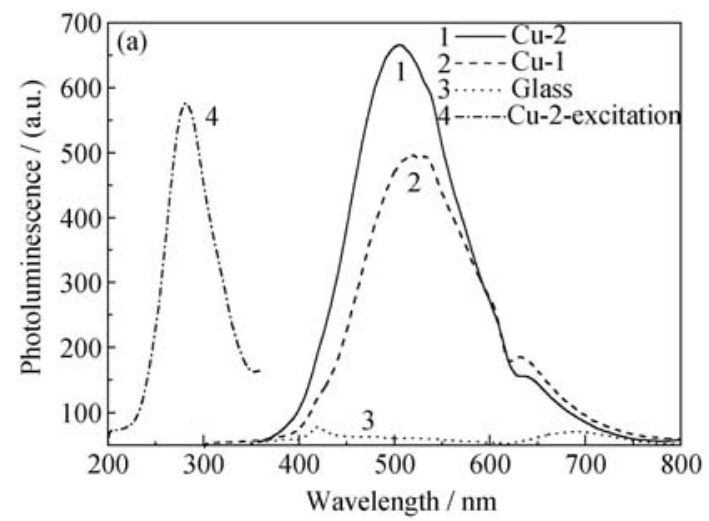

$\mathrm{Cu}-3$ 。将样品 $\mathrm{Cu}-1$ 在 $\mathrm{H}_{2}$ 气氛下 $400^{\circ} \mathrm{C}$ 热处理 $15 \mathrm{~min}$ 和 $30 \mathrm{~min}$ 得到样品 $\mathrm{Cu}-\mathrm{H}-1$ 和 $\mathrm{Cu}-\mathrm{H}-2$ 。将钠钻硅酸 盐玻璃浸入 $0.05 \mathrm{wt} \%$ 的 $\mathrm{AgNO}_{3}$ 与 $\mathrm{NaNO}_{3}$ 混合熔盐中 $350^{\circ} \mathrm{C}$ 下分别离子交换 2、24 和 $168 \mathrm{~h}$ 得到样品 $\mathrm{Ag}-1$ 、 $\mathrm{Ag}-2$ 和 Ag-3。将钠钙硅酸盐玻璃分别浸入含 $0.1 \mathrm{wt} \%$ 和 $0.2 \mathrm{wt} \% \mathrm{AgNO}_{3}$ 的 $\mathrm{NaNO}_{3}$ 熔盐中 $350^{\circ} \mathrm{C}$ 离子交换 168 $h$ 得到样品 Ag-4、和 Ag-5。将样品 Ag-2 在 $\mathrm{H}_{2}$ 气氛 下 $350^{\circ} \mathrm{C}$ 热处理 $0.5 \mathrm{~h}$ 得到 $\mathrm{Ag}-6$ 。将硅酸盐玻璃浸 入 $0.05 \mathrm{wt} \%$ 的 $\mathrm{AgNO}_{3}$ 与 $\mathrm{NaNO}_{3}$ 混合熔盐中 $350^{\circ} \mathrm{C}$ 离 子交换 $168 \mathrm{~h}$, 取出后自然冷却，清洗晾干; 然后将该 样品放入 $\mathrm{H}_{2}$ 气氛炉中 $350^{\circ} \mathrm{C}$ 热处理 $10 \mathrm{~min}$, 随炉冷却 后再将该样品浸入 $\mathrm{CuCl}$ 熔盐中, $450^{\circ} \mathrm{C}$ 离子交换 $3 \mathrm{~h}$ 得到样品 $\mathrm{Ag}-\mathrm{Cu}-1$ 。样品 $\mathrm{Ag}-\mathrm{Cu}-1$ 进一步在 $400^{\circ} \mathrm{C}$ $\mathrm{H}_{2}$ 热处理 $15 \mathrm{~min}$ 得到样品 $\mathrm{Ag}-\mathrm{Cu}-2$ 。用 970CRT 苂 光分光光度计测量样品的光致发光性能。

\section{2 结果与讨论}

图1给出了离子交换时间和离子交换温度对样 品光致发光的影响。由图1可见，钠钙硅酸盐玻璃在 $280 \mathrm{~nm}$ 光激发下未出现发光峰, 而离子交换样品在 绿光范围出现明显发光峰, 其中样品 $\mathrm{Cu}-1$ 的发光峰 位于 $520 \mathrm{~nm}$ 附近; 样品 $\mathrm{Cu}-2$ 的发光峰位于 $505 \mathrm{~nm}$ 附 近且比样品 $\mathrm{Cu}-1$ 发光峰更强; 样品 $\mathrm{Cu}-3$ 的发光峰位 于514 nm附近, 其发光峰强度明显低于样品 $\mathrm{Cu}-2$ 的 发光峰。这些发光峰起源于 $\mathrm{Cu}^{+}$的 $3 \mathrm{~d}^{9} 4 \mathrm{~s}^{1}$ 到 $3 \mathrm{~d}^{10}$ 之间 电子跃迁。根据Laporte规则, 自由 $\mathrm{Cu}^{+}$的 $3 \mathrm{~d}^{9} 4 \mathrm{~s}^{1}$ 与 $3 \mathrm{~d}^{10}$ 之间电子跃迁是禁止的, 在玻璃中 $\mathrm{Cu}^{+}$与玻璃中 的 $\mathrm{O}$ 形成畸变的八面体配位, 造成 $\mathrm{Cu}^{+}$周围的局域电 场非对称性, $3 \mathrm{~d}^{9} 4 \mathrm{~s}^{1}$ 与 $3 \mathrm{~d}^{10}$ 之间电子跃迁不再被禁止， 其激发与发射原理见图 $2^{[26]}$ 。

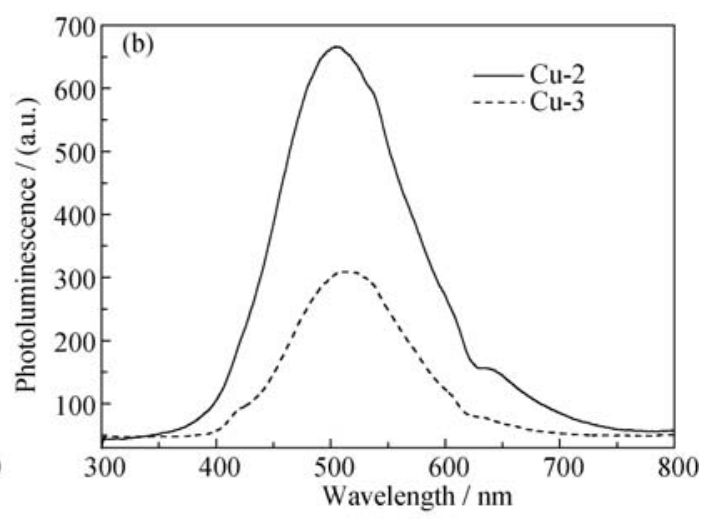

图 1 离子交换时间(a)和离子交换温度(b)对样品光致发光的影响

Fig. 1 Influences of ion-exchanged period (a) and temperature (b) on photoluminescence of $\mathrm{Cu}$-doped silicate glasses PL spectra excited by $280 \mathrm{~nm}$ and PLE spectra detected by $500 \mathrm{~nm}$ 


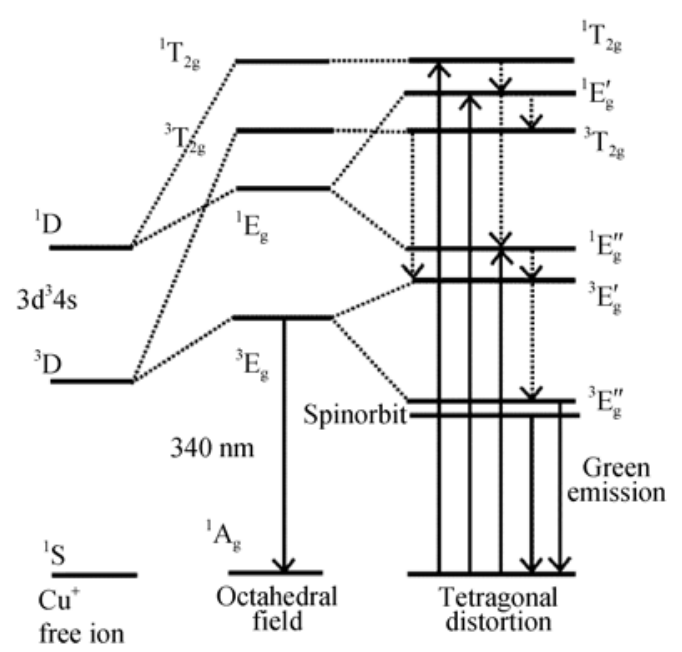

图 2 玻璃中 $\mathrm{Cu}^{+}$的八面体配位场能级图 ${ }^{[26]}$

Fig. 2 Level configuration scheme for the $\mathrm{Cu}^{+}$ion, with the effect of a tetragonal distortion of the octahedral field ${ }^{[26]}$

$\mathrm{Cu}^{+}$与配体相互作用, 造成 $3 \mathrm{~d}^{9} 4 \mathrm{~s}$ 激发态分裂成 ${ }^{1} \mathrm{~T}_{2 \mathrm{~g}} 、{ }^{3} \mathrm{~T}_{2 \mathrm{~g}} 、{ }^{1} \mathrm{E}_{\mathrm{g}}$ 和 ${ }^{3} \mathrm{E}_{\mathrm{g}}$, 并且三重态在自旋一轨道相互 作用下可以进一步分裂成其它亚能级。特别指出的 是, 最低能级 ${ }^{3} \mathrm{E}_{\mathrm{g}}$ 分裂成两个自旋-轨道能级 $\mathrm{T}_{1 \mathrm{~g}}$ 和 $\mathrm{T}_{2 \mathrm{~g}}, 500 \sim 520 \mathrm{~nm}$ 的发光峰起源于三重态能级 $\mathrm{T}_{1 \mathrm{~g}}$ 和 $\mathrm{T}_{2 \mathrm{~g}}$ 到基态的跃迁。在碱金属卤化物基质中, $[\mathrm{CuX}]_{6}$ 八面体的最低三重态位于 $340 \mathrm{~nm}$ 。由于玻璃中的 $[\mathrm{CuO}]_{6}$ 八面体发生四角畸变, 在 $280 \mathrm{~nm}$ 激发波长下, 发射波长向绿光区域漂移。这种畸变造成 ${ }^{3} \mathrm{E}_{\mathrm{g}}$ 能级进 一步分裂成 ${ }^{3} E_{\mathrm{g}}{ }^{\prime}$ (进一步分裂成自旋-轨道部分) 和 ${ }^{3} E_{\mathrm{g}}{ }^{\prime}$, 可以显著降低三重态的发射能量。

离子交换时间越长, 玻璃中 $\mathrm{Cu}^{+}$含量越多, 导致 样品 $\mathrm{Cu}-2$ 比 $\mathrm{Cu}-1$ 的发光强度强。离子交换过程如下:

$$
-\mathrm{Si}-\mathrm{O}-\mathrm{Na}+\mathrm{Cu}^{+} \stackrel{\mathrm{T}}{\longrightarrow}-\mathrm{Si}-\mathrm{O}-\mathrm{Cu}+\mathrm{Na}^{+}
$$

(1) 发光峰位随着离子交换时间的延长而蓝移, 这 主要是由 $\mathrm{Cu}^{+}$在玻璃中的配位结构畸变不同所引起 的。样品 $\mathrm{Cu}-2$ 和 $\mathrm{Cu}-3$ 离子交换时间相同，随着离 子交换温度的升高, 发光峰强度明显降低, 这是由于 高温离子交换会导致玻璃中出现 $\mathrm{Cu}^{2+}\left(2 \mathrm{Cu}^{+}=\mathrm{Cu}^{2+}+\mathrm{Cu}\right)$, $\mathrm{Cu}^{+}$的激发态电子将部分能量传递给 $\mathrm{Cu}^{2+}$, 或者 $\mathrm{Cu}^{2+}$ 和配体 $\mathrm{O}^{2-}$ 之间发生电荷迁移, 造成 $\mathrm{Cu}^{+}$的发光强度显 著降低 ${ }^{[27]}$ 。

图 3 给出了样品 $\mathrm{Cu}-\mathrm{H}-1$ 和 $\mathrm{Cu}-\mathrm{H}-2$ 的光致发光 谱。由图 3 可以看出, 经过 $\mathrm{H}_{2}$ 热处理后, 样品的发 光峰几乎消失, 发光峰强度随着热处理时间的延长 而降低。这说明随着热处理的进行, 玻璃中 $\mathrm{Cu}^{+}$浓 度快速降低，即发生如下反应：

$\equiv \mathrm{Si}-\mathrm{O}-\mathrm{Cu}+\frac{1}{2} \mathrm{H}_{2} \stackrel{\mathrm{T}}{\longrightarrow} \equiv \mathrm{Si}-\mathrm{O}-\mathrm{H}+\mathrm{Cu}$

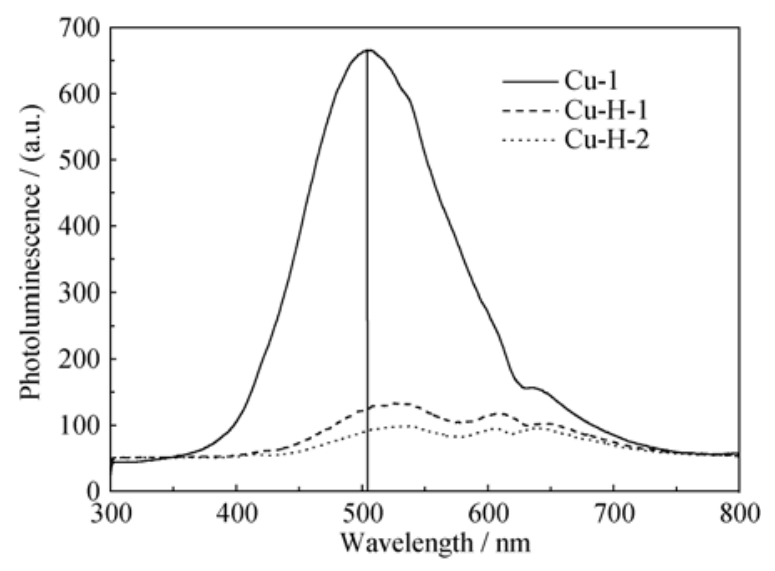

图 3 在 $280 \mathrm{~nm}$ 激发光下样品 $\mathrm{Cu}-\mathrm{H}-1$ 和 $\mathrm{Cu}-\mathrm{H}-2$ 的光致发 光谱

Fig. 3 PL spectra of samples $\mathrm{Cu}-\mathrm{H}-1$ and $\mathrm{Cu}-\mathrm{H}-2$ excited by $280 \mathrm{~nm}$

$n \mathrm{Cu} \stackrel{\text { Nucleation }}{\longrightarrow} \mathrm{Cu}_{n} \stackrel{\text { Growth }}{\longrightarrow} \mathrm{Cu}$ nanoparticle

由图 1 和图 3 可知, 硅酸盐玻璃中的 $\mathrm{Cu}^{+}$除了 发绿光外, 在 $640 \mathrm{~nm}$ 附近存在一个弱的发光峰, 该发光峰与玻璃中的非桥氧 $\equiv \mathrm{Si}-\mathrm{O}^{-}$发光中心有 关 ${ }^{[28]}$ 。

图 4 为样品 $\mathrm{Ag} 1 、 \mathrm{Ag} 2 、 \mathrm{Ag} 3$ 在 $240 \mathrm{~nm}$ 的光激 发下的发射光谱。由图 4(a)可知，在 $240 \mathrm{~nm}$ 激发光 下钠钻硅酸盐玻璃在 300 600 nm 出现一个宽而弱 的发光峰, 这个发光峰起源于 $\mathrm{L}-$ 色心 $(\equiv \mathrm{Si}-\mathrm{O}-\mathrm{Na})^{[29]}$, 离子交换不同时间后，样品均呈现两个很宽的发光 峰, 分别位于 $300 \sim 600 \mathrm{~nm}$ 和 620 780 nm 之间。随 着离子交换时间的延长, 样品的发光强度增加。由 图 4(b)显示, 300 600 nm 的宽峰可拟合成 3 个峰, 分 别位于 $354 、 398$ 和 $458 \mathrm{~nm}$ 。图 4(a)的激发谱显示, 通 过监测样品 Ag-1 在 $360 \mathrm{~nm}$ 处的发射光谱, 获得最 佳激发光波长为 $250 \mathrm{~nm}$, 此波长来源于 $\mathrm{Ag}^{+}$的 $4 \mathrm{~d}^{10} \rightarrow 4 \mathrm{~d}^{9} 5 \mathrm{~S}^{1}$ 跃迁。当 $\mathrm{Ag}^{+}-\mathrm{Na}^{+}$离子交换后, $\mathrm{Ag}^{+}$与钠 钙硅酸盐玻璃中的非桥氧结合形成畸变的八面体配 位，其能级图类似于图 2。354 nm 附近的紫光发光 峰起源于 ${ }^{3} \mathrm{E}_{\mathrm{g}}$ 到基态的跃迁, $398 \mathrm{~nm}$ 附近的发光峰 起源于 ${ }^{3} \mathrm{E}_{\mathrm{g}}$ 到基态的跃迁, $458 \mathrm{~nm}$ 附近的发光峰起 源于 $\mathrm{Ag}_{2}{ }^{+}$才簇 ${ }^{[30-33]}$ 。离子交换时间越长, 交换进入 玻璃中的 $\mathrm{Ag}^{+}$越多, 其发光强度越强, 即发光强度 顺序为 $\mathrm{Ag}-3(\mathrm{PL})>\mathrm{Ag}-2(\mathrm{PL})>\mathrm{Ag}-1(\mathrm{PL})$ 。图中 620 780 nm 左右处的峰与玻璃中的非桥氧 $\equiv \mathrm{Si}^{-} \mathrm{O}^{-}$发 光中心有关, 而 $\equiv \mathrm{Si}-\mathrm{O}^{-}$发光中心很大程度上与玻璃 中的杂质和缺陷有关 ${ }^{[28]}$ 。在离子交换过程中, 离子 的交换和团聚会形成缺陷，所以离子交换时间越长， 离子交换和团聚程度越高，引起的缺陷相对增多， 

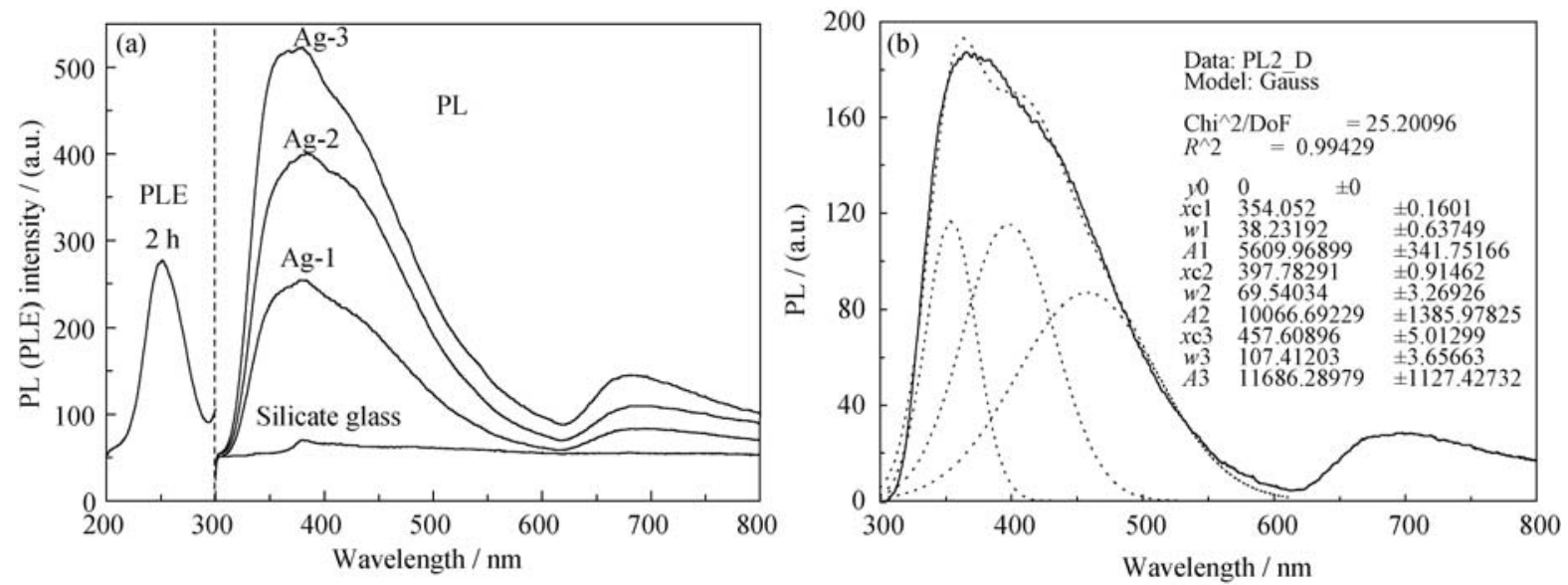

图 4 (a)在 $240 \mathrm{~nm}$ 激发波长下掺 $\mathrm{Ag}$ 钠钻硅酸盐玻璃的发光谱, (b)Ag-1 发光谱减去硅酸盐玻璃基体的发光谱，点线为高斯 拟合

Fig. 4 (a) PL spectra of Ag-doped silicate glasses excited by $240 \mathrm{~nm}$ and a representative excitation spectrum detected by $360 \mathrm{~nm}$ emission; (b) PL differential spectrum of sample Ag-1 subtracting sodalime silicate glass (solid line) and the corresponding Gaussian fits (dot lines)

其发光强度也越强。

图 5 给出了熔盐中 $\mathrm{AgNO}_{3}$ 质量分数对样品发射 光谱的影响。由图可知, 随着 $\mathrm{AgNO}_{3}$ 质量分数的增 加，样品的发光强度减弱，发光峰位有一定的红移。 这是因为 $\mathrm{AgNO}_{3}$ 质量分数提高后，进入玻璃中的 $\mathrm{Ag}^{+}$易聚集或被还原, 形成 $\mathrm{Ag}^{+}-\mathrm{Ag}^{+}$和 $\mathrm{Ag}^{0}-\mathrm{Ag}^{+}$等结 构, 它们发光强度均低于孤立的 $\mathrm{Ag}^{+}$的发光强度, 但发光峰位随 $\mathrm{Ag}^{+}$团簇尺寸的增大而红移。

图 6 给出了样品 $\mathrm{Ag}-2$ 和 $\mathrm{Ag}-6$ 的光致发光谱和 激发谱。从图 6(a) 可知, 在 $240 \mathrm{~nm}$ 紫外光激发下, 样 品 Ag-2 和 Ag-6 的光致发光谱有明显改变。样品 Ag-2 在 300 600 nm 范围有一强且宽的发光峰, 经 过 $\mathrm{H}_{2}$ 热处理后, 样品 Ag-6 仅在 345、510、670 nm 附近出现弱的发光峰。这是由于在 $\mathrm{H}_{2}$ 中热处理导致

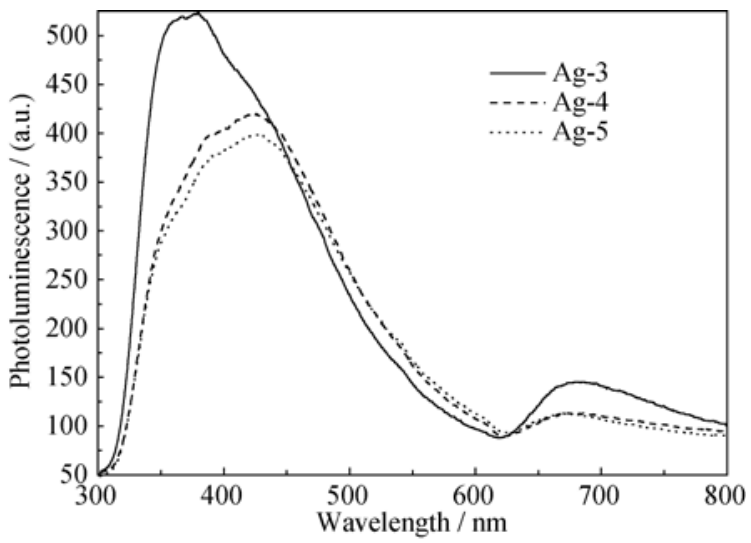

图 $5 \mathrm{AgNO}_{3}$ 质量分数对掺 $\mathrm{Ag}$ 硅酸盐玻璃光致发光的影响 Fig. 5 Influences of $\mathrm{AgNO}_{3}$ mass fraction on photoluminescence of Ag-doped silicate glasses at excitation wavelength of $240 \mathrm{~nm}$
玻璃中的 $\mathrm{Ag}^{+}$被还原成中性 $\mathrm{Ag}$ 原子，银原子与玻璃 中的银离子结合, 生成银团簇, 从而造成玻璃中 $\mathrm{Ag}^{+}$浓度快速降低。银团簇的发光强度均低于孤立 的 $\mathrm{Ag}^{+}$的发光强度, 所以样品 $\mathrm{Ag}-6$ 的发光峰强度显 著降低。玻璃中的银离子在氢气气氛热处理过程中, 发生如下反应:

$$
\begin{gathered}
\equiv \mathrm{Si}-\mathrm{O}-\mathrm{Na}+\mathrm{Ag}^{+} \stackrel{\mathrm{T}}{\longrightarrow} \equiv \mathrm{Si}-\mathrm{O}-\mathrm{Ag}+\mathrm{Na}^{+} \\
\equiv \mathrm{Si}-\mathrm{O}-\mathrm{Ag}+\frac{1}{2} \mathrm{H}_{2} \stackrel{\mathrm{T}}{\longrightarrow} \equiv \mathrm{Si}-\mathrm{O}-\mathrm{H}+\mathrm{Ag} \\
\mathrm{Ag}+\mathrm{Ag}^{+} \rightarrow \mathrm{Ag}_{2}^{+} \\
\mathrm{Ag}^{+}+\mathrm{Ag}^{+} \rightarrow \mathrm{Ag}_{2}^{2+} \\
\mathrm{Ag}_{2}^{+}+\mathrm{Ag} \rightarrow \mathrm{Ag}_{3}^{+} \\
\mathrm{Ag}_{2}^{2+}+\mathrm{Ag} \rightarrow \mathrm{Ag}_{3}^{2+} \\
\mathrm{Ag}_{3}^{2+}\left(\mathrm{Ag}_{3}^{+}\right)_{\text {growth }}^{\longrightarrow}
\end{gathered}
$$

图 6(b)、(c)和(d)显示, 监测 $345 \mathrm{~nm}$ 的发光峰, 发现 Ag-6 最佳激发峰值位于 $250 \mathrm{~nm}$ 左右。由以上 讨论可知, $345 \mathrm{~nm}$ 处的发光峰是由 ${ }^{3} \mathrm{E}_{\mathrm{g}}{ }^{\prime}$ 到基态的跃 迁所引起的, 说明样品经过 $\mathrm{H}_{2}$ 热处理后仍有 $\mathrm{Ag}^{+}$ 存在，但其浓度有限，所以其发光峰强度显著降 低。510 nm 左右处的发光峰与 $\mathrm{Ag}_{3}{ }^{2+}$ 有关 ${ }^{[34-36]}$ 。监 测 $510 \mathrm{~nm}$ 的发光峰, 发现两个最佳激发峰: 326 和 $290 \mathrm{~nm}$, 其中 $326 \mathrm{~nm}$ 为主激发峰, 明显不同于孤 立 $\mathrm{Ag}^{+}$的吸收 $(220 \sim 280 \mathrm{~nm})$, 这也间接证明 $510 \mathrm{~nm}$ 的发光峰不是由畸变的 $\left[\mathrm{AgO}_{6}\right]$ 八面体引起的, 而 是由 $\mathrm{Ag}_{3}{ }^{2+}$ 引起的。670 $\mathrm{nm}$ 左右的发光峰较 $\mathrm{H}_{2}$ 热 处理前有一定的降低, 表明 $\mathrm{H}_{2}$ 热处理有利于降低 玻璃中的缺陷浓度。 

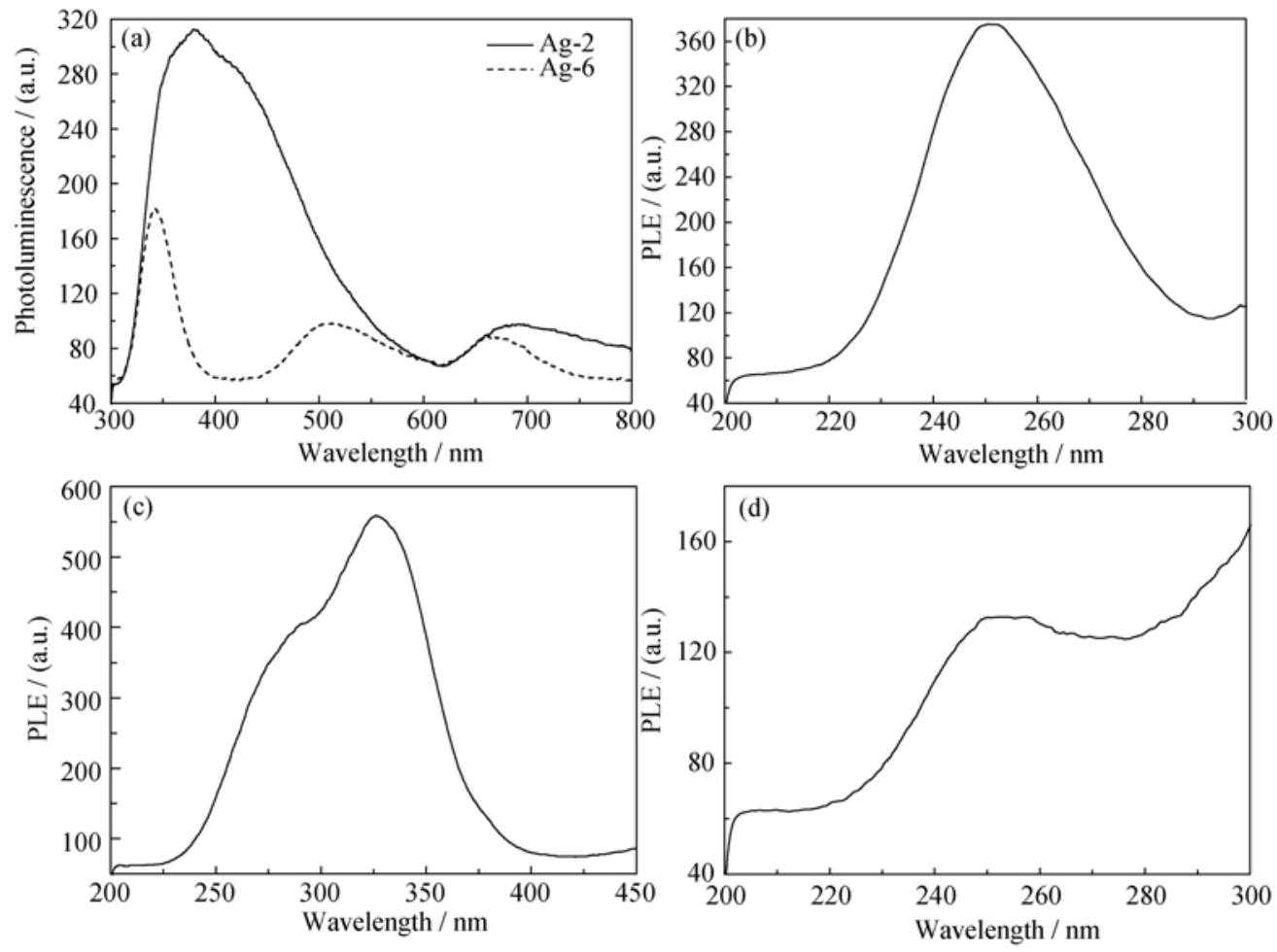

图 6 样品 Ag-2 和 Ag-6 的发光谱和发射谱

Fig. 6 PL and PLE spectra of samples Ag-2 and Ag-6

(a) PL spectra excited by $240 \mathrm{~nm}$; (b-d) PLE spectra of Ag-6 detected by $345 \mathrm{~nm}, 510 \mathrm{~nm}$ and $670 \mathrm{~nm}$, respectively

图 7 给出了样品 $\mathrm{Ag}-\mathrm{Cu}-1$ 和 $\mathrm{Ag}-\mathrm{Cu}-2$ 的发光谱 和发射谱。由图 7(a)可知, 二次离子交换样品在 $240 \mathrm{~nm}$ 光激发下, 分别在 336、372 和 $600 \mathrm{~nm}$ 附近出现发光峰, 其中 $600 \mathrm{~nm}$ 处的发光峰为主要发光峰, 其它两个为弱 发光峰。如前所述, 336 和 $372 \mathrm{~nm}$ 发光峰分别来自 于 $\mathrm{Ag}^{+}$的 ${ }^{3} \mathrm{E}_{\mathrm{g}}{ }^{\prime}$ 和 ${ }^{3} \mathrm{E}_{\mathrm{g}}$ 到基态的跃迁, $600 \mathrm{~nm}$ 附近的发 光峰可分解成两个峰, 分别位于 551 和 $631 \mathrm{~nm}$, 其中 $551 \mathrm{~nm}$ 附近的发光峰起源于 $\mathrm{Cu}^{+}$的三重态能 级 $\mathrm{T}_{1 \mathrm{~g}}$ 和 $\mathrm{T}_{2 \mathrm{~g}}$ 到基态的跃迁, $631 \mathrm{~nm}$ 附近的发光峰 起源于玻璃中的非桥氧 $\equiv \mathrm{Si}-\mathrm{O}^{-}$。样品 $\mathrm{Ag}-\mathrm{Cu}-1$ 的 发光强度远低于样品 $\mathrm{Cu}-1$, 同时, 发光峰位发生
红移，表明样品 $\mathrm{Ag}-\mathrm{Cu}-1$ 中的 $\mathrm{Cu}^{+}$浓度远低于样品 $\mathrm{Cu}-1$, 这是由于随着反应(4) (10)的进行, 玻璃中 的 $\equiv \mathrm{Si}-\mathrm{O}-\mathrm{Na}$ 键被 $\equiv \mathrm{Si}-\mathrm{O}-\mathrm{H}$ 取代, 同时在样品表面 生成 $\mathrm{Ag}$ 纳米颗粒。银纳米颗粒阻止熔盐中 $\mathrm{Cu}^{+}$ 向玻璃扩散, 同时, $\mathrm{Cu}^{+}$与玻璃中 $\mathrm{H}^{+}$的离子交换 也很困难。进一步 $\mathrm{H}_{2}$ 热处理, 导致样品 $\mathrm{Ag}-\mathrm{Cu}-2$ 中的发光峰几乎消失, 表明样品 $\mathrm{Ag}-\mathrm{Cu}-2$ 中的 $\mathrm{Cu}^{+} 、 \mathrm{Ag}^{+}$基本被还原。监测样品 $\mathrm{Ag}-\mathrm{Cu}-1$ 在 $550 \mathrm{~nm}$ 和 $640 \mathrm{~nm}$ 处的激发谱, 发现最佳激发波长均为 $331 \mathrm{~nm}$, 其中 $318 \mathrm{~nm}$ 处的激发峰为发射峰 $640 \mathrm{~nm}$ 的倍频。
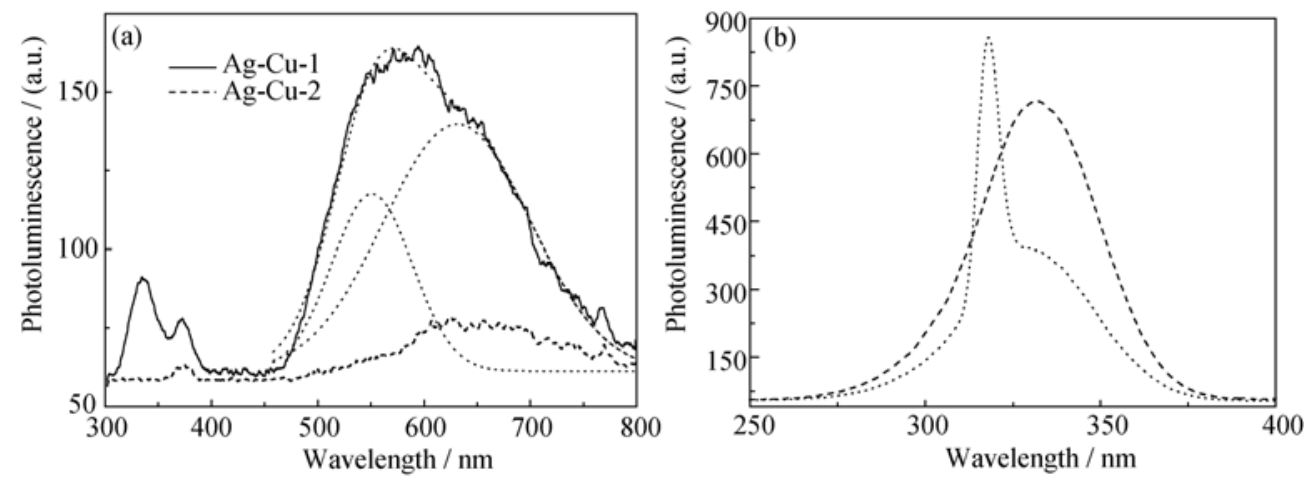

图 7 样品 $\mathrm{Ag}-\mathrm{Cu}-1$ 和 $\mathrm{Ag}-\mathrm{Cu}-2$ 的发光谱和激发谱

Fig. 7 PL and PLE spectra of samples Ag-Cu-1 and Ag-Cu-2

(a) PL spectra excited by $240 \mathrm{~nm}$ (b) PLE spectra of sample Ag-Cu-1 detected by $550 \mathrm{~nm}$ (dash curve) and $640 \mathrm{~nm}$ (dot curve), respectively 


\section{3 结论}

掺 $\mathrm{Ag}^{+}\left(\mathrm{Cu}^{+}\right)$硅酸盐玻璃的发光峰强度随离子交 换时间的延长而增强, 随熔盐中 $\mathrm{AgNO}_{3}$ 含量的提高 而降低。 $\mathrm{H}_{2}$ 中热处理后, 发光峰强度迅速降低甚至 消失, 新出现的 $510 \mathrm{~nm}$ 左右处的发光峰与 $\mathrm{Ag}_{3}{ }^{2+}$ 有 关。掺 $\mathrm{Ag}^{+}$硅酸盐玻璃的发光峰位于 354、398 和 $458 \mathrm{~nm}$, 分别起源于 ${ }^{3} \mathrm{E}_{\mathrm{g}}$ 到基态的跃迁, ${ }^{3} \mathrm{E}_{\mathrm{g}}$ 到基态 的跃迁和 $\mathrm{Ag}_{2}{ }^{+}$才簇。掺 $\mathrm{Cu}^{+}$硅酸盐玻璃的发光峰位 于 500-520 nm 范围内, 起源于三重态能级 $\mathrm{T}_{1 \mathrm{~g}}$ 和 $\mathrm{T}_{2 \mathrm{~g}}$ 到基态的跃迁。 $\mathrm{Cu}^{2+}$ 的存在造成 $\mathrm{Cu}^{+}$的发光强度显 著降低。波长大于 $600 \mathrm{~nm}$ 的发光峰与玻璃中的非 桥氧缺陷有关。掺 $\mathrm{Ag}^{+}$硅酸盐玻璃经 $\mathrm{H}_{2}$ 热处理后, 再经过第二次离子交换往玻璃中掺 $\mathrm{Cu}^{+}$是十分困 难的。

\section{参考文献:}

[1] KREIBIG U, VOLLMER M. Optical Properties of Metal Clusters. Berlin: Springer, 1995. 300.

[2] FARADAY M. The Bakerian Lecture: On the relations of gold and other metals to light. Proceedings of the Royal Society of London, 8(8):356-361.

[3] DOERINGg W E, NIE S M. Single-molecule and single-nanoparticle SERS: Examining the roles of surface active sites and chemical enhancement J. Phys. Chem. B, 2002, 106(2):311-317.

[4] CAMDEN J P, DIERINGER J A, ZHAO J, et al. Controlled plasmonic nanostructures for surface-enhanced spectroscopy and sensing. Acc. Chem. Res., 2008, 41(12): 1653-1661.

[5] HAES A J, DUYNE R P V. A nanoscale optical biosensor: sensitivity and selectivity of an approach based on the localized surface plasmon resonance spectroscopy of triangular silver nanoparticles. J. Am. Chem. Soc., 2002, 124(35):10596-10604.

[6] RASCHKE G, KOWARIK S, FRANZL T, et al. Biomolecular recognition based on single gold nanoparticle light scattering. $\mathrm{Na}$ no Lett., 2003, 3(7): 935-938.

[7] JAIN P K, HUANG X H, EL-SAYED I H, et al. Noble metals on the nanoscale: optical and photothermal properties and some applications in imaging, sensing, biology, and medicine. Acc. Chem. Res., 2008, 41(12):1578-1586.

[8] COTTER D, MANNING R J, BLOK K J, et al. Nonlinear optics for high-speed digital information processing. Science, 1999, 286(5444): 1523-1528.

[9] EATON D F. Nonlinear optical materials. Science, 1991, 253(5017):281-287.

[10] WANG W, YANG G, CHEN Z, et al. Iron nanoparticles in amor- phous $\mathrm{BaTiO}_{3}$ thin films with large third-order optical nonlinearity. Journal of Applied Physics, 2002, 92(12):7242-7245

[11] DONG Z W, YANG X C, LI Z H, et al. Ultrafast dynamics of copper nanoparticles embedded in soda-lime silicate glass fabricated by ion exchange. Thin Solid Films, 2009, 517(21): 6046-6049.

[12] YANG X C, LI Z H, LI W J, et al. Optical nonlinearity and ultrafast dynamics of ion exchanged silver nanoparticles embedded in soda-lime silicate glass. Chinese Science Bulletin, 2008, 53(5): 695-699.

[13] YANG X C, DONG Z W, LIU H X, et al. Effects of thermal treatment on the third-order optical nonlinearity and ultrafast dynamics of $\mathrm{Ag}$ nanoparticles embedded in silicate glasses. Chemical Physics Letters, 2009, 475(4/5/6): 256-259.

[14] YANG X C, LI W J, LI Z H, et al. Nonlinear optical properties of $\mathrm{Ag}$ nanoparticles/glass composites measured on a femtosecond time scale. Journal of Wuhan University of Technology-Mater. Sci. Ed., 2007, 22(Suppl.): 69-71.

[15] TERVONEN A, WEST B R, HONKANEN S. Ion-exchanged glass waveguide technology: a review. Optical Engineering, 2011, 50(7): $71107-71114$

[16] GONELlA F, MAZZOLDI P. Metal Nanocluster Composite Glasses, in H. S. Nalwa(Ed), Handbook of Nanostrured Materials and Nanotechnology. New York: Academic Press, 2000: 81.

[17] YANG X C, DU T L, CHEN S, et al. Forming mechanism of Ag nanoparticles in silicate glasses by optical spectroscopy. Journal of Functional Materials \& Devices, 2006, 12(3):177-181.

[18] YANG X C, XU J X, LI Z H, et al. The formation mechanism of $\mathrm{Cu}$ nanoparticles and local structures of copper atoms in silicate glass. Journal of the Chinese Ceramic Society, 2008, 36(7): 990-993.

[19] XU J X, YANG X C, LIU H X, et al. The formation process of $\mathrm{Ag}-\mathrm{Cu}$ nanoparticles in silicate glass. Journal of the Chinese Ceramic Society, 2009, 37(6): 953-959.

[20] LIU H X, YANG X C, ZHAO J F, et al. The absorption spectra of silver nanoparticles/silicate glass composite materials. Journal of the Chinese Ceramic Society, 2010, 38(10):1922-1926.

[21] ZHAO J F, YANG X C, HUANG M, et al. Local structure of $\mathrm{Ag}-\mathrm{Cu}$ nano-particles in composite materials. Journal of the Chinese Ceramic Society, 2012, 40(2):312-316.

[22] YANG X C. Influences of preparation conditions on the formation and depth dispersion of Ag nanoparticles in soda-lime silicate glass. Journal of Non-Crystalline Solids, 2015, 430(15): 87-93.

[23] YANG X C, LI L L, HUANG M, et al. In situ synthesis of Ag-Cu bimetallic nanoparticles in silicate glass by a two-step ion-exchange route. Journal of Non-Crystalline Solids, 2011, 357(11/12/13): 2306-2308.

[24] YANG X C, LI W J, Dubiel M, et al. Silver structure environments 
in ion-exchanged silicate glasses studied by x-ray absorption fine structure. J. Nanosci. Nanotech., 2009, 9(2):1659-1662.

[25] YANG X C, LI W J, LI Z H, et al. Depth profiles of Ag nanoparticles in silicate glass. Applied Physics A, 2008, 90(3):465-467.

[26] BORSELlA E, VECCHIO A D, GARCIA M A, et al. Copper doping of silicate glasses by the ion-exchange technique: A photoluminescence spectroscopy study. Journal of Applied Physics, 2002, 91(1): 90-98.

[27] TANAKA K, YANO T, SHIBATA $\mathrm{S}$, et al. $\mathrm{Cu}^{+}$-doped $\mathrm{CaO}-\mathrm{P}_{2} \mathrm{O}_{5}$ glasses for lasers. J. Non-Crystalline Solids, 1994, 178(3): 9-14.

[28] PODLIPENSKY A V, GREBENEV V, SEIFERT G, et al. Ionization and photomodification of Ag nanoparticles in soda-lime glass by 150 fs laser irradiation: a luminescence study. Journal of Luminescence, 2004, 109(3/4): 135-142.

[29] EiChelbaum M, RADEMANN K, HOELl A, et al. Photoluminescence of atomic gold and silver particles in soda-lime silicate glasses. Nanotechnology, 2008,19(13): 135701-135710.

[30] BORSELLA E, BATTAGLIN G, GARCIA M A, et al. Structural incorporation of silver in soda-lime glass by the ion-exchange process: a photoluminescence spectroscopy study. Appl. Phys. A:
Mater. Sci. Proocess, 2000, 71(2): 125-132.

[31] MANIKANDANA D, MOHANC S, NAIR K G M. Absorption and luminescence of silver nanocomposite soda-lime glass formed by $\mathrm{Ag}^{+}-\mathrm{Na}^{+}$ion-exchange. Materials Research Bulletin, 2003, 38(9/10):1545-1550.

[32] VERON O, BLONDEAU P J, ABDELKRIM N, et al. Luminescence study of silver nanoparticles obtained by annealed ionic exchange silicate glasses. Plasmonics, 2010, 5(2):213-219.

[33] BORSELLA E, GONELLA F, MAZZODI P, et al. Spectroscopic investigation of silver in soda-lime glass. Chemical Physics Letters, 1998, 284(5/6):429-434.

[34] HE F, LI Q T, ZHANG Z Y. The photoluminescence properties of float glass doped with siliver. Journal of the Chinese Ceramic Society, 2003, 31(7) : 711-714.

[35] PAJE S E, GARCIA M A, LIOPIS J, et al. Optical spectroscopy of silver ion-exchanged As-doped glass. Journal of Non-Crystalline Solids, 2003, 318(3):239-247.

[36] WANG H Y, YE S, LIU T H, et al. Molecular-like Ag clusters and Eu ${ }^{3+}$ co-sensitized efficient broadband spectral modification with enhanced $\mathrm{Yb}^{3+}$ emission. J. Am. Ceram. Soc., 2016, 99(7): 2376-2381. 